\title{
Role of the Alarmin S100A9 protein in inducing Achilles tendinopathy in rats
}

\author{
Bin-An Zhao ${ }^{1 \#}$, Jun $\mathrm{Li}^{1 \#}$, Chao Xue ${ }^{1}$, Jie $\mathrm{Li}^{2}$, Heng-An Ge${ }^{1}$, Biao Cheng ${ }^{1}$, Qi-Hang Su${ }^{1}$ \\ ${ }^{1}$ Department of Orthopedics, Shanghai Tenth People's Hospital, School of Medicine, Tongji University, Shanghai, China; ${ }^{2}$ Department of \\ Orthopedics, Shanghai Zhabei District Central Hospital, Shanghai, China \\ Contributions: (I) Conception and design: BA Zhao, J Li, C Xue, HA Ge, QH Su; (II) Administrative support: None; (III) Provision of study materials \\ or patients: None; (IV) Collection and assembly of data: BA Zhao; (V) Data analysis and interpretation: J Li, C Xue, HA Ge, QH Su; (VI) Manuscript \\ writing: All authors; (VII) Final approval of manuscript: All authors. \\ \#These authors contributed equally to this work. \\ Correspondence to: Qihang Su, PhD; Biao Cheng, MD, PhD, Prof. Department of Orthopedics, Shanghai Tenth People's Hospital, School of \\ Medicine, Tongji University, No. 301 Yanchang Middle Road, Shanghai 200072, China. Email: 15221378017@163.com; drbiaocheng@163.com.
}

Background: This study aimed to investigate the correlation between the Alarmin S100A9 protein and Achilles tendinopathy (AT), and to reveal the role of this protein in inducing AT.

Methods: In this study, 40 male Sprague-Dawley rats were randomly divided into four groups: Control group (received no treatment), Injury group (Achilles tendon tissues were cut intraoperatively), S100A9 group (received a subcutaneous injection of rhS100A9 solution), and S100A9 + Paquinimod group [received a subcutaneous injection of rhS100A9 and Paquinimod (1:1 ratio) into the Achilles tendon]. At 1 week postoperatively, the four groups of rats were euthanized, and the Achilles tendon tissues were isolated for histological staining, immunohistochemistry (IHC), immunofluorescence, Sirius Red (SR) staining, and terminal deoxynucleotidyl transferase-mediated dUTP-biotin nick end labeling assay.

Results: Compared with both the Control and S100A9 + Paquinimod groups, the Injury and S100A9 groups exhibited higher expression levels of S100A9 protein, matrix metalloproteinase-3 (MMP-3), and inflammatory factors. Regarding histomorphology [hematoxylin-eosin (HE) staining and Safranin O/fast green (SOFG; fast green and Safranin) training], the Achilles tendon tissues in the Injury and S100A9 groups showed AT-like changes, and the fibers were extremely disorderly, non-bundled, and ruptured, and some nuclei were spindles. As for collagen (Col) remodeling, a large number of fresh collagen fibers had formed, the amounts of Col-I and Col-II were lower, and a large quantity of Col-III was present. Additionally, a large number of tendon cells had died in both the Injury and S100A9 groups.

Conclusions: This study showed that Alarmin S100A9 can induce AT-like morphological changes and local inflammatory reactions, trigger collagen fiber remodeling and tendon cell apoptosis, and ultimately induce AT.

Keywords: Alarmin S100A9; Achilles tendinopathy (AT); inflammation; degeneration; animal experiment

Submitted Oct 09, 2021. Accepted for publication Nov 19, 2021.

doi: $10.21037 / \mathrm{atm}-21-5945$

View this article at: https://dx.doi.org/10.21037/atm-21-5945

\section{Introduction}

Achilles tendinopathy (AT) is a common cause of disability in many athletes due to the continuous prolonged intense functional demands imposed on the Achilles tendon (1). Symptomatic AT often causes localized pain, swelling, and dysfunction around tendons (2), thus severely affecting the daily life of people or the careers of athletes. However, the 
etiology of AT is not presently clear; therefore, symptomatic treatments including analgesia, anti-inflammatory medication, and functional rehabilitation training are commonly applied in clinical practice, and surgery is usually only performed if the Achilles tendon is damaged or ruptured (3). There are numerous studies relating to the pathogenesis of AT, including inflammation, micro-injury, oxidative stress, and degeneration (4-8). Current research on inflammation tends to focus on changes in the extracellular matrix (ECM). Research has shown that the overload and use of the Achilles tendon causes local inflammation or micro-damage, which in turns leads to changes in the extracellular microenvironment and the production of matrix metalloproteinases (MMPs). This triggers the reconstruction of collagen fibers (the main composition changes from type I collagen to type III collagen), the formation of microvessels, and the causes degeneration of numerous tendon cells, thus resulting in AT (9).

Recent research into inflammation-related musculoskeletal diseases has begun to investigate alarmins, which are substances that exist in humans and animals that are able to trigger inflammation-like reactions in the body, such as those observed when tissues/cells are damaged. Subsequently, this reaction enhances the immune defense abilities of the body, or can aggravate the immunopathological damage on the body, and also facilitates the repair and regeneration processes within organs and tissues. Alarmins include the S100s family, interleukins, heat shock proteins, and high mobility group proteins (10-13). Multiple studies have highlighted that the Alarmin S100A9 protein can induce or aggravate osteoarthritis (OA) by activating inflammatory reactions (14-16). Zhang et al. (17) showed that the S100A9 protein is also highly expressed in the murine rotator cuff tendinopathy model. Crowe et al. (18) revealed that the S100A8/S100A9 protein exhibits high expression levels in human supraspinatus tendinopathy cases, which exerts a crucial effect in the pathological processes of tendinopathy. However, the pathophysiological relationship between AT and Alarmin S100A9, as well as the histological characteristics of AT, have rarely been researched.

Therefore, in this study, a rat Achilles tendon injury model and S100A9 induction model were constructed in order to evaluate the morphological changes of rat Achilles tendon tissues, inflammation, collagen fiber remodeling, and tendon cell apoptosis based on histological and cytological investigations, which revealed pathophysiological relationships between AT and Alarmin S100A9. In order to initiate or speed up the development of AT, it was assumed that Alarmin S100A9 was more highly expressed in injured Achilles tendon tissues, thus providing a new potential target for AT treatment. We present the following article in accordance with the ARRIVE reporting checklist (available at https://dx.doi.org/10.21037/atm-21-5945).

\section{Methods}

\section{Animal models and ethics}

In this study, 40 specific pathogen free-grade male SpragueDawley rats (Shanghai SLAC Laboratory Animal Co., Ltd., Shanghai, China; aged 10-12 weeks old, average weight 250-300 g) were used. The rats were randomly divided into four groups: normal control group (Control group, $n=10$ ), Achilles tendon injury group (Injury group, $n=10$ ), a group that received S100A9 injected into the Achilles tendon (S100A9 group, $\mathrm{n}=10$ ), and a group that received both the inhibitor S100A9 and Paquinimod via injection into the Achilles tendon (S100A9 + Paquinimod group, $\mathrm{n}=10$ ) (19). Sampling and histological analysis were conducted at 1 week postoperatively (20).

Experiments were performed under a project license (SHSY-IEC-BG/05.08/04.0) granted by the Ethics Committee of Shanghai Tenth People's Hospital (Tenth People's Hospital Affiliated to Tongji University) Medical Ethics Committee and in compliance with the institutional guidelines for the care and use of animals. A protocol was prepared before the study without registration.

\section{Model preparation}

The rats were anesthetized via an intraperitoneal injection of $100 \mathrm{mg} / \mathrm{kg}$ ketamine $+8 \mathrm{mg} / \mathrm{kg}$ xylazine (Sangon Biotech, Shanghai, China) (21), and underwent surgery following deep anesthesia. The Control group received no treatment. In the Injury group, the left Achilles tendons of the rats were located by palpation, and incision and dissection were used to expose the Achilles tendon tissues. Thereafter, the tissue was dissected from the middle of Achilles tendon, prior to the skin being sutured and disinfected. In the S100A9 group, $0.5 \mathrm{~mL}$ of $1 \mathrm{mg} / \mathrm{mL}$ rhS100A9 solution was injected subcutaneously into the Achilles tendon in the left foot of each rat. In the S100A9 + Paquinimod group, $0.25 \mathrm{~mL}$ of $1 \mathrm{mg} / \mathrm{mL}$ rhS100A9 solution (Abcam, Shanghai, China) and $0.25 \mathrm{~mL}$ of $1 \mathrm{mg} / \mathrm{mL}$ Paquinimod solution (MedChemExpress Co., Ltd., Shanghai, China) were injected subcutaneously into 


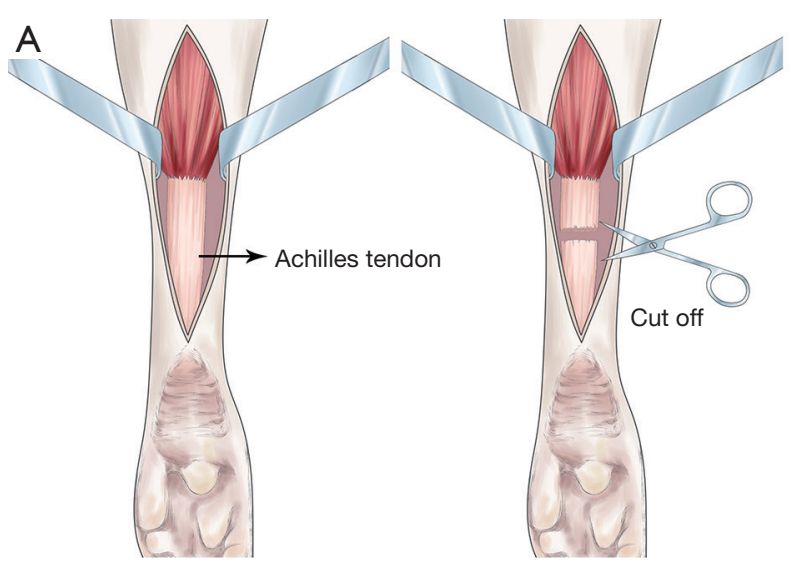

Injury group

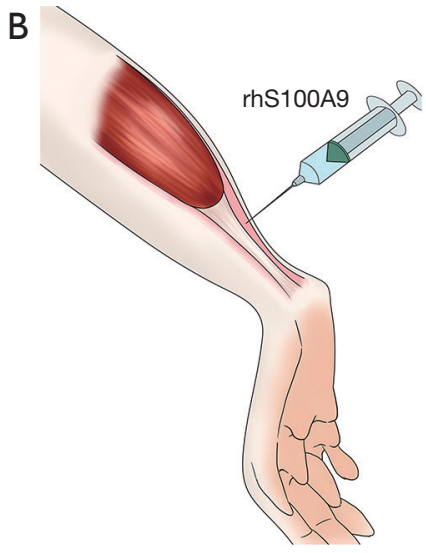

S100A9 group

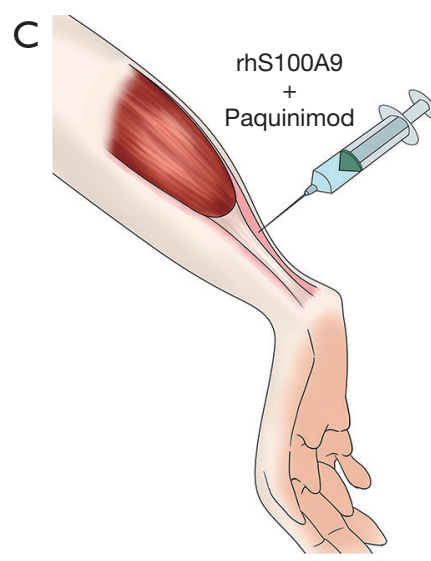

S100A9 + Paquinimod group

Figure 1 Modeling process of experimental animals. (A) The Achilles tendon of rats was located and dissected so that it was exposed. The tendon was then cut in the middle section, and the skin was then sewn together and disinfected (Injury group). (B) The rat Achilles tendon was located and subcutaneously injected with $0.5 \mathrm{~mL}$ of $1 \mathrm{mg} / \mathrm{mL}$ rhS100A9 solution (S100A9 group). (C) The rat Achilles tendon was located and subcutaneously injected with $0.5 \mathrm{~mL}$ of $1: 1 \mathrm{mixed}$ solution of $1 \mathrm{mg} / \mathrm{mL} \mathrm{rhS} 100 \mathrm{~A} 9 \mathrm{and} 1 \mathrm{mg} / \mathrm{mL}$ Paquinimod (S100A9 + Paquinimod group).

the Achilles tendon in the left foot of each rat (Figure 1) $(19,22,23)$.

\section{Tissue preparation}

At 1 week postoperatively, the four groups of rats were anesthetized and culled by cervical dislocation. The Achilles tendon tissues from the operation site were separated out and extracted. These tissues were fixed with $4 \%$ paraformaldehyde, dehydrated, embedded in paraffin, and then sectioned $(4 \mu \mathrm{m})$. Finally, the tissue sections were dehydrated, deparaffinized, and stored on glass slides at room temperature.

\section{Histological staining}

In this study, three kinds of non-specific histological staining techniques were performed on the Achilles tendon tissues: hematoxylin-eosin (HE) staining, Safranin O/ fast green (SOFG; fast green and Safranin) staining, and Masson's trichrome staining (Wuhan Servicebio Technology Co., Ltd., China). According to the conventional protocols (24-26), the prepared tissue sections were stained with the corresponding staining solutions and photomicrographs were obtained. In order to review the Masson's staining, Image-Pro Plus 6.0 software (Media Cybernetics, Silver Spring, USA) was applied to analyze the proportions of fresh and immature collagen fibers within the Achilles tendon tissues.

\section{Immunobistochemistry (IHC)}

In this study, S100A9, collagen-I (Col-I), Col-II, and MMP-3 were analyzed by IHC according to routine published procedures (26): (I) the paraffin-embedded tissues sections were rehydrated; (II) antigen retrieval was conducted in citric acid ( $\mathrm{pH}$ 6.0) antigen retrieval buffer; (III) the endogenous peroxidase activity was blocked using $3 \%$ hydrogen peroxide; (IV) incubation in $3 \%$ bovine serum albumin Blocking Buffer; (V) tissues were embedded in individual primary antibodies (S100A9/Col-I/ColII:Phosphate buffer saline $=1: 100$, Servicebio, Wuhan, China), and the sections were placed flat in a wet box and incubated overnight at $4{ }^{\circ} \mathrm{C}$; (VI) the sections were then incubated in the secondary antibodies and subjected to a diaminobenzidine (DAB) chromogenic reaction; (VII) the nuclei were counterstained with hematoxylin stain solution; (VIII) dehydration and coverslip mounting were performed; and (IX) the stained images with under differing magnifications were obtained using a scanning imaging system and light microscopy (Nikon DS-U3, Nikon, Japan), and Image-Pro Plus 6.0 software was utilized to measure the average optical density (AOD) of each index, so as to quantify the expression of different indexes. 


\section{Sirius Red (SR) staining}

In this study, SR staining was conducted to evaluate the composition ratio of Col-I and Col-III in the four groups of Achilles tendon tissues. Specifically, the paraffin-embedded slices were deparaffinized, washed with water, dyed in SR staining solution for $8 \mathrm{~min}$, dehydrated, and the coverslips were mounted. Thereafter, the tissue sections underwent microscopic examination and image acquisition using a polarization microscope (NIKON ECLIPSE E100, Nikon, Japan), and the composition ratios of Col-I (coarse orange or bright red fibers) and Col-III (fine green fibers) were analyzed using Image-Pro Plus 6.0 software (27).

\section{Immunofluorescence (IF)}

In this study, interleukin-6 (IL-6) and tumor necrosis factor- $\alpha$ (TNF- $\alpha$ ) were detected using IF. Paraffin-embedded sections were deparaffinized, rehydrated, and then subjected to antigen retrieval. Subsequently, primary antibodies (IL-6 or TNF- $\alpha$ antibody, Servicebio, Wuhan, China) and secondary antibodies were added independently for incubation. Afterwards, 4'6-diamidino-2-phenylindole (DAPI) was used to stain the nucleus, tissue autofluorescence was quenched, coverslips were mounted, and photomicrographs were taken under a fluorescence microscope (NIKON ECLIPSE C1, Nikon, Japan) (28). Ultimately, the average fluorescence intensity was quantified with $\mathrm{AOD}$, so as to quantify the expression of different indexes.

\section{Terminal deoxynucleotidyl transferase dUTP nick end labeling (TUNEL) assay}

TUNEL is a detection method for evaluating apoptosis (28). Paraffin-embedded sections were deparaffinized and rehydrated. Following antigen retrieval using proteinase $\mathrm{K}$, the membrane was broken using the membrane breaking solution. Following equilibrium at room temperature, the reaction solution provided within the TUNEL kit (terminal deoxynucleotidyl transferase:Cy3-dUTP:buffer =1:5:50, G1501, Servicebio, Wuhan, China) was added. Next, the nuclei were stained with DAPI and the slides coverslipped. Finally, the images were collected using a fluorescence microscope, and the positive apoptotic cell rate (red fluorescence) was calculated.

\section{Statistical analysis}

All data in this study were analyzed using SPSS software
(Version 2.0, IBM Corp., Armonk, New York, USA). P<0.05 represented a statistically significant difference. The fresh collagen ratio (Masson's staining) and the AOD of IHC (for S100A9, Col-I, Col-II, and MMP-3) and IF (for IL-6 and TNF- $\alpha$ ) indexes, Col-III ratio (using SR staining), and apoptosis rate (using TUNEL) did not conform to variance homogeneity; therefore, they were statistically analyzed via Kruskal-Wallis tests. The remaining data were subjected to descriptive statistics.

\section{Results}

\section{Tissue morphology and structure}

Following HE and SOFG staining, the tissue morphology and structure of the four groups of Achilles tendon tissues were analyzed (Figure 2). The HE staining results (Figure 2A) revealed that collagen fibers in the Control group were arranged in an orderly manner, tightly and continuously in bundles, the number of tendon cells was moderate, and the nucleus grew fusiform. In the Injury group, the fibrous structure of the ruptured end of the Achilles tendon was disorderly and not bundled, there were ruptured fibers, and some nuclei were not fusiform. In the S100A9 group, the fibrous tissues were disorderly, presenting in a small wave shape, were not bundled, and some ruptured fibers were present. However, in the S100A9 + Paquinimod group, the tissue characteristics were the same as those in the Control group, and the fibers were bundled and arranged neatly.

SOFG staining results also illustrated the above morphological characteristics, although the contrast between the groups was more obvious. In addition, there were red staining components amongst the fibrous tissues in the Injury and S100A9 groups, revealing that the ECM or fibrous components differed and showed cartilage matrix-like red staining, which seemed to be the precursor of heterotopic ossification of Achilles tendon tissues (29) (Figure 2B).

\section{Fresh collagen formation}

Masson's staining can display the morphological characteristics as shown in HE staining, and can also show fresh and immature collagen fibers (blue) (30). We found (Figure $3 A$ ) that a large number of fresh and immature collagen fibers formed in rats from both the Injury and S100A9 groups. The fibers in the Injury group were concentrated mainly in the ruptured end of the Achilles tendon, whilst those in the S100A9 group 

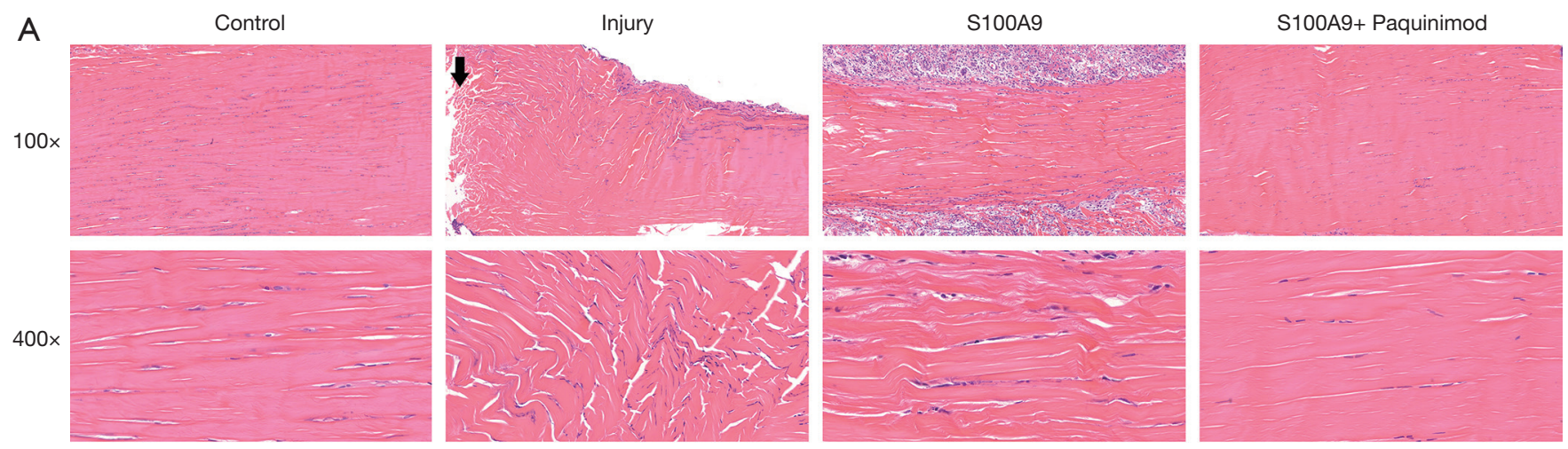

B

Control
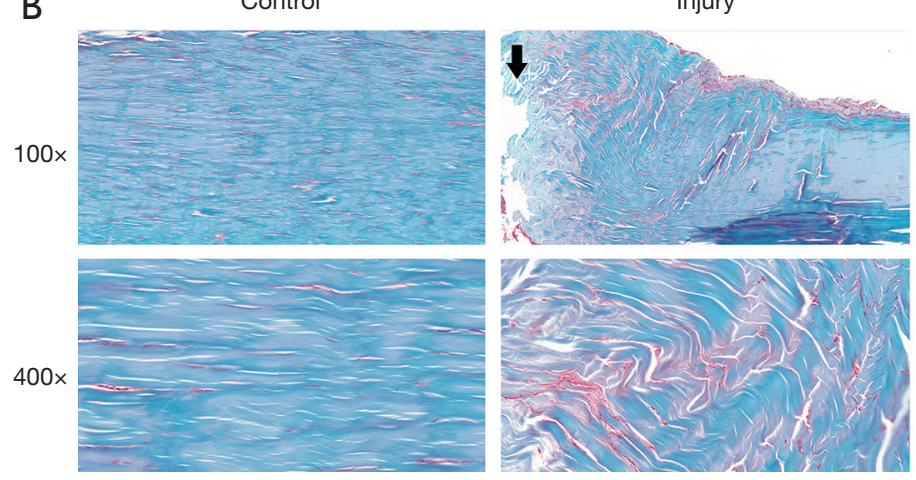

S100A9
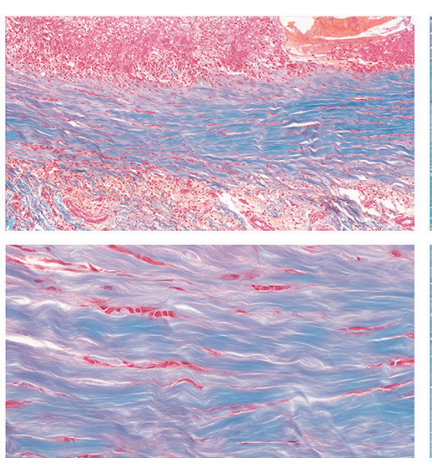

S100A9+ Paquinimod
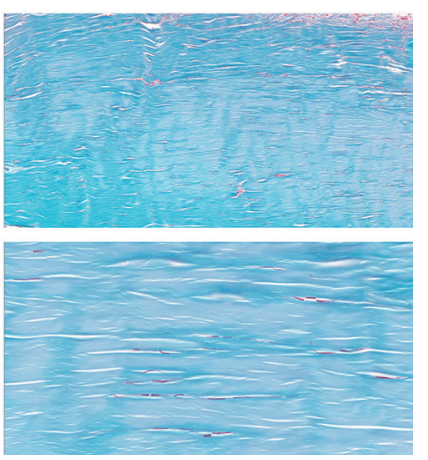

Figure 2 Non-specific histological staining showed that Achilles tendon fibers in the Injury and S100A9 groups were highly disorderly and not bundled, and ruptured fibers were present. (A) hematoxylin-eosin (HE) staining. (B) Safranin O/fast green (SOFG) staining. Black arrow: position of the ruptured end of Achilles tendon.

were evenly distributed among mature fibers. In the S100A9 + Paquinimod group, relatively less fresh collagen fibers had formed. Moreover, it was observed from the statistical analysis results (Figure $3 B$ ) that the area ratio of fresh collagen fibers in the Injury and S100A9 groups were $17.20 \% \pm 0.99 \%$ and $14.09 \% \pm 2.67 \%$, respectively, whereas these figures were lower in the Control group $(1.75 \% \pm 0.50 \%)$ and the S100A9 + Paquinimod group $(2.55 \% \pm 0.83 \%)$, and the differences were statistically significant [Control-Injury, $\mathrm{H}=-4.983, \mathrm{P}<0.05$, ControlS100A9: $\mathrm{H}=-3.778, \mathrm{P}<0.05$, (S100A9 + Paquinimod)Injury: $\mathrm{H}=3.873, \mathrm{P}<0.05$, (S100A9 + Paquinimod)-S100A9: $\mathrm{H}=2.668, \mathrm{P}<0.05]$. However, there were no significant differences in the area ratio of fresh collagen fibers between the Control and S100A9 + Paquinimod groups [Control(S100A9 + Paquinimod): $\mathrm{H}=-1.109, \mathrm{P}=0.267]$.

\section{S100A9 expression level}

S100A9 IHC results (Figure 4) demonstrated that compared to the Control and S100A9+Paquinimod groups, the S100A9 protein was more highly expressed in the Injury and S100A9 groups [Control-Injury: $\mathrm{H}=-4.039$, $\mathrm{P}<0.05$, Control-S100A9: $\mathrm{H}=-4.613, \mathrm{P}<0.05$, (S100A9 + Paquinimod)-Injury: $\mathrm{H}=3.044, \mathrm{P}=0.002$, (S100A9 + Paquinimod)-S100A9: $\mathrm{H}=3.618, \mathrm{P}<0.05]$. There were no significant differences in S100A9 expression levels between the Injury and S100A9 groups (Injury-S100A9: $\mathrm{H}=-0.574$, $\mathrm{P}=0.566)$.

\section{Composition of collagen types}

Figures $5 A, 5 B$ display the IHC results pertaining to Col-I and Col-II, respectively. We observed that normal Achilles tendon tissues were mainly comprised of Col-I, with relatively low Col-II content. At the same time, statistical analysis results (Figure 5C) showed that the content of Col-I and Col-II declined in the Injury and S100A9 groups, which differed significantly from both the Control and S100A9+ Paquinimod groups $(\mathrm{P}<0.05)$. 

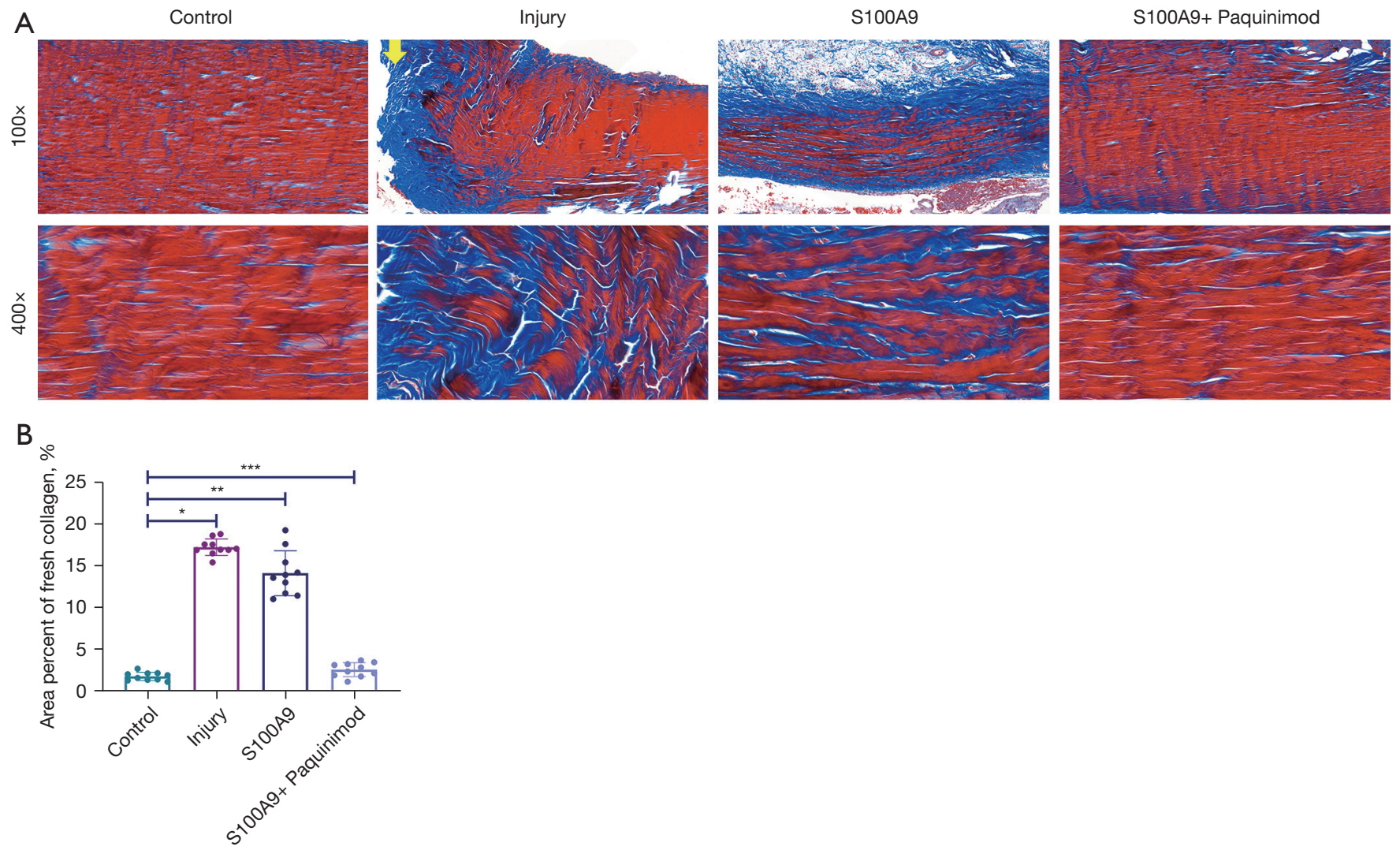

Figure 3 Masson's trichrome staining revealed collagen formation. (A) Masson's trichrome staining displayed that numerous fresh and immature collagen fibers were produced (blue) in the Injury and S100A9 groups. (B) Statistical chart of the area ratio of blue fresh collagen fibers. Yellow arrow: position of the ruptured end of an Achilles tendon. *, Control-Injury: H=-4.983, P<0.05; **, Control-S100A9: H=3.778, $\mathrm{P}<0.05 ;{ }^{* * *}$, Control-(S100A9 + Paquinimod): $\mathrm{H}=-1.109, \mathrm{P}=0.267$.

The polarized light images (Figure 5D) displaying SR staining showed the ratio of Col-III in Achilles tendon tissues in the four groups: Col-I was visualized as coarse orange or bright red fibers and Col-III was displayed as fine green fibers. It was observed that a large amount of ColIII formed at the ruptured end of the Achilles tendons in rats in the Injury group, while the newly-formed Col-III in the S100A9 group was mainly distributed among Col-I fibers. Col-I was still the main collagen fiber component in the Control and S100A9 + Paquinimod group animals. Additionally, the ratio of Col-III in the Injury and S100A9 groups was markedly higher than that in either the Control or S100A9 + Paquinimod groups $(\mathrm{P}<0.05$; Figure $5 E)$.

\section{ECM and inflammation}

MMP-3 was highly expressed in the Injury and S100A9 groups (Figure 6A). It was primarily concentrated within the ruptured end in the Injury group tendons, but was evenly distributed among tendon fiber tissues in the S100A9 group, indicating that the extracellular microenvironment of the two groups had changed significantly. IF results (Figure 6B,6C) revealed the expression patterns of the inflammatory factors IL- 6 and TNF- $\alpha$. It was observed that numerous inflammatory factors were released in the Injury and S100A9 groups, and the expression of TNF- $\alpha$ appeared to be higher than that of IL-6.

Moreover, the statistical analysis results displayed that MMP-3 (Figure 6D), IL-6, and TNF- $\alpha$ (Figure $6 E$ ) in the Injury and S100A9 groups were higher than those in either the Control or S100A9 + Paquinimod groups $(\mathrm{P}<0.05)$.

\section{Apoptosis}

The TUNEL assay (Figure $7 A$ ) demonstrated that compared with the apoptosis levels observed in the Control 
group $(3.82 \% \pm 1.78 \%)$ or the S100A9 + Paquinimod group $(7.53 \% \pm 1.71 \%)$, the apoptosis rate in the Injury group $(20.90 \% \pm 4.89 \%)$ and the S100A9 group $(20.40 \% \pm 5.20 \%)$ was notably higher, and these differences were statistically significant (Figure $7 B, \mathrm{P}<0.05$ ).

\section{Discussion}

Through comparative study of a Control group, Injury group, S100A9 group, and S100A9 + Paquinimod group, it was found that the S100A9 protein was abundantly

A
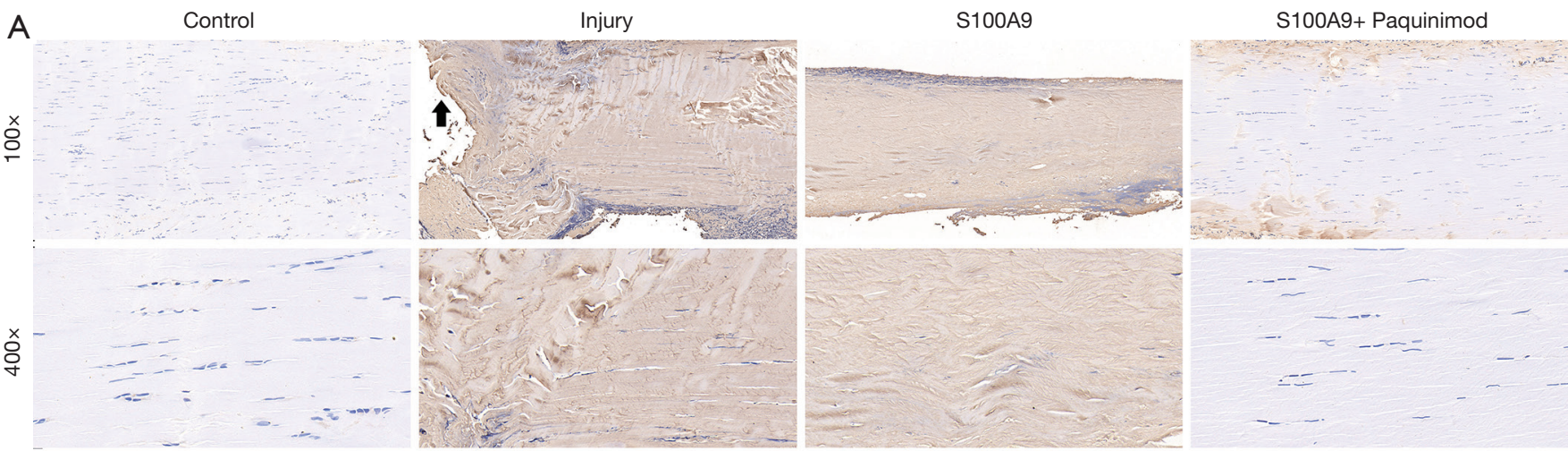

B

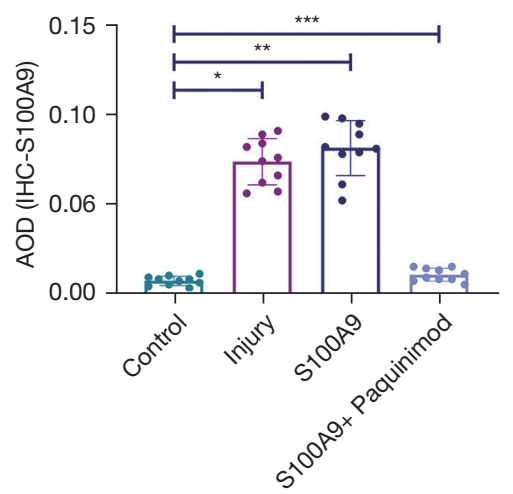

Figure 4 S100A9 immunohistochemistry expression. (A) Immunohistochemical results showing how S100A9 manifested; S100A9 was highly expressed in the Injury and S100A9 groups. Black arrow: Position of the ruptured end of an Achilles tendon. (B) Statistical chart of the AOD of S100A9 immunohistochemical expression. *, Control-Injury: H=-4.039, $\mathrm{P}<0.05$; **, Control-S100A9: H=-4.613, $\mathrm{P}<0.05$; ***, Control(S100A9 + Paquinimod): $\mathrm{H}=-0.995, \mathrm{P}=0.320$.
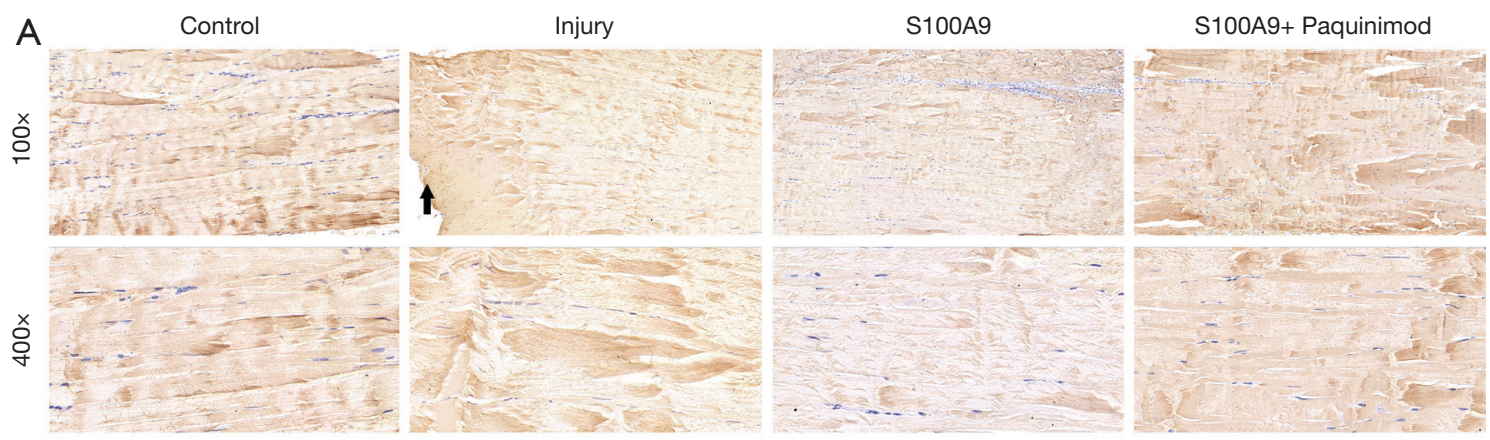


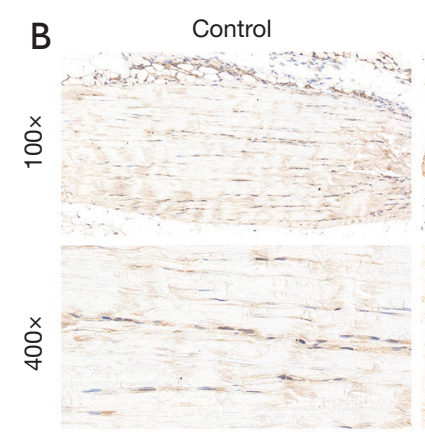

C

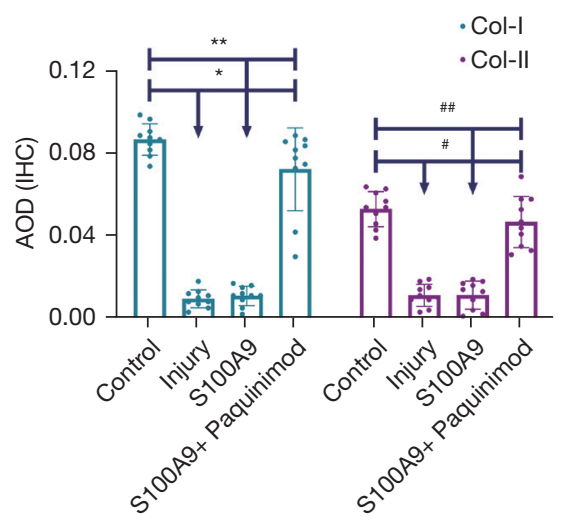

D
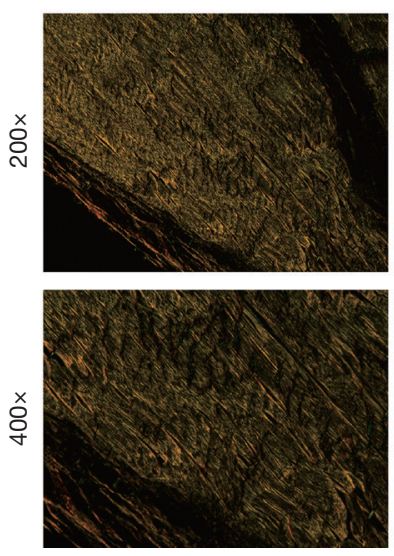

Injury

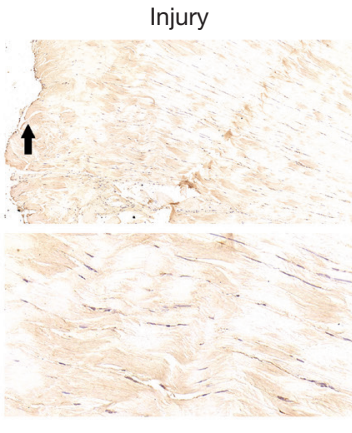

Col-II

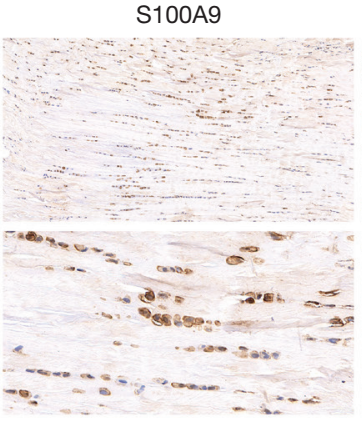

S100A9+ Paquinimod

$\mathrm{E}$

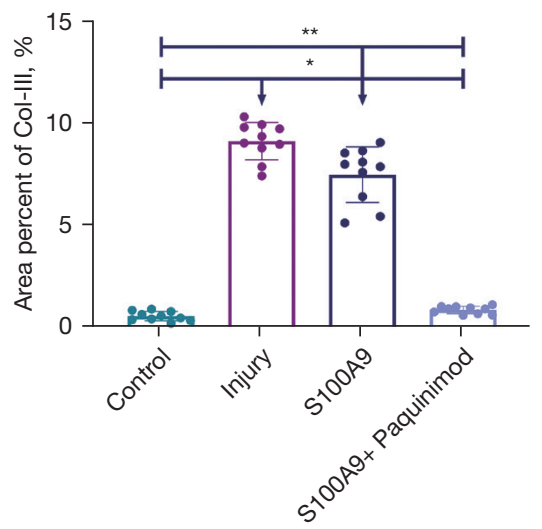

S100A9
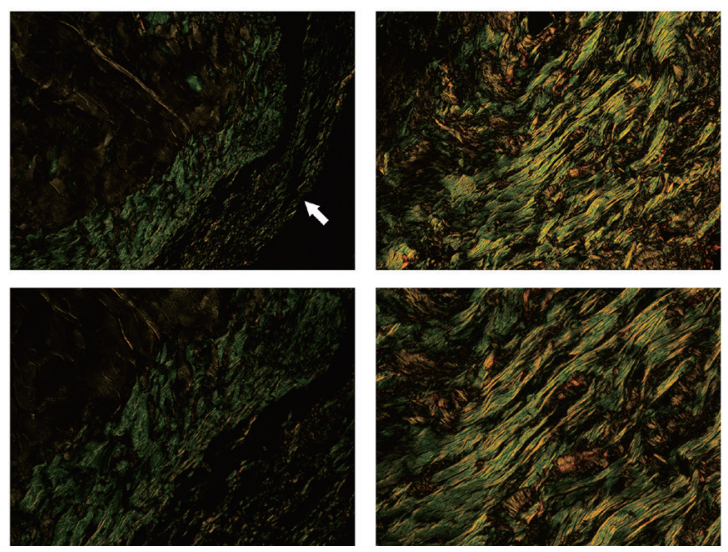

S100A9+ Paquinimod
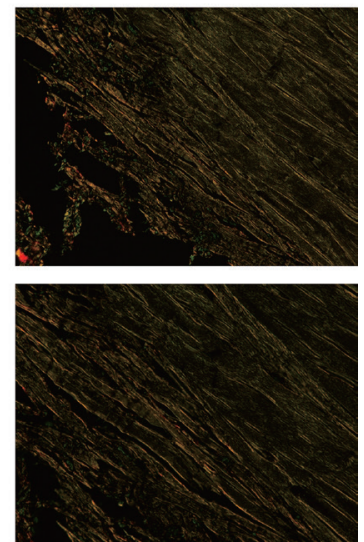

Figure 5 Collagen I and II immunohistochemistry. (A) Col-I IHC. (B) Col-II IHC. Black arrow: Position of the ruptured end of Achilles tendon. (C) Statistical chart of AOD of the IHC expressions of Col-I and Col-II: *, Injury-Control (or S100A9 + Paquinimod): H=4.556 (or -3.503), $\mathrm{P}<0.05$; **, S100A9-Control (or S100A9 + Paquinimod): $\mathrm{H}=4.154$ (or -3.101), $\mathrm{P}<0.05$; ${ }^{*}$, Injury-Control (or S100A9 + Paquinimod): $\mathrm{H}=4.153$ (or -3.540), $\mathrm{P}<0.05$; \#, S100A9-Control (or S100A9 + Paquinimod): $\mathrm{H}=4.114$ (or -3.502), $\mathrm{P}<0.05$. (D) Polarized light image of SR staining. White arrow: the ruptured end of Achilles tendon; Col-I was coarse orange or bright red fiber, and Col-III was the fine green fiber. (E) Statistical chart of the area ratio of Col-III in the polarized light image: *, Injury-Control (or S100A9 + Paquinimod): H=-5.174 (or 3.797), $\mathrm{P}<0.05$; **, S100A9-Control (or S100A9 + Paquinimod): $\mathrm{H}=-3.854$ (or 2.477), $\mathrm{P}<0.05$. 

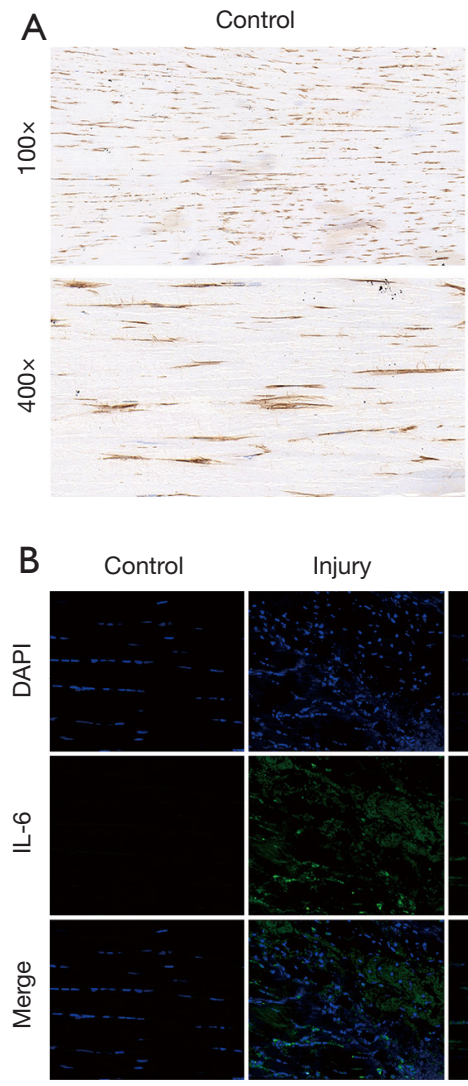

$400 \times$

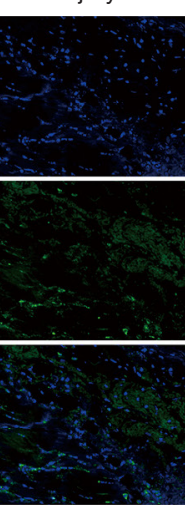

$400 x$
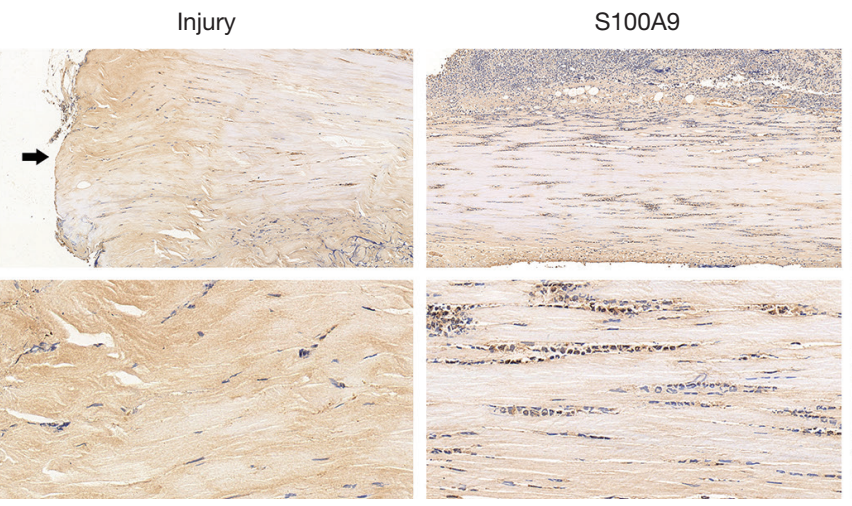

S100A9+ Paquinimod

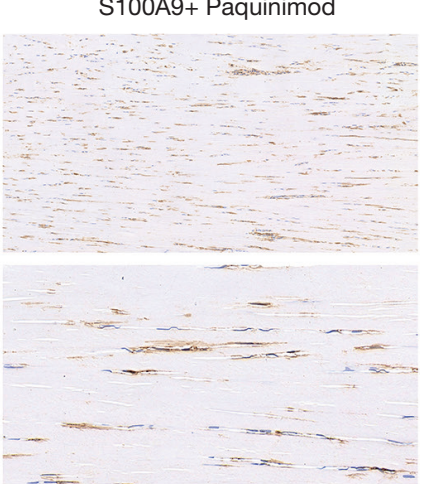

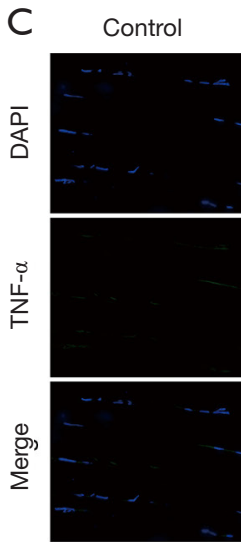

$400 \times$

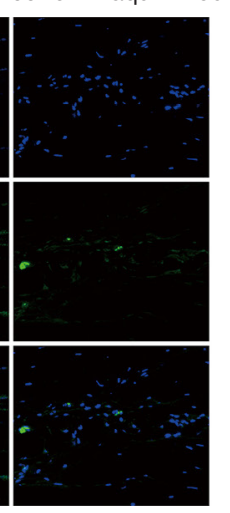

$400 x$

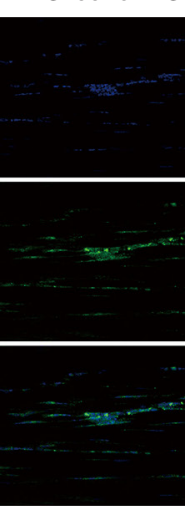

$400 \times$
$\mathrm{D}$

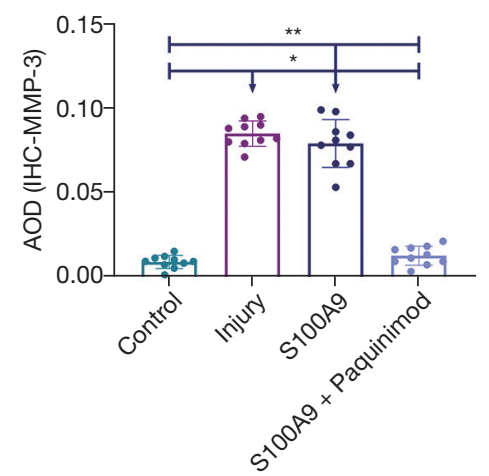

$E$

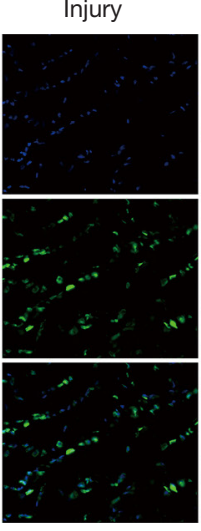

$400 x$
S100A9 S100A9 + Paquinimod

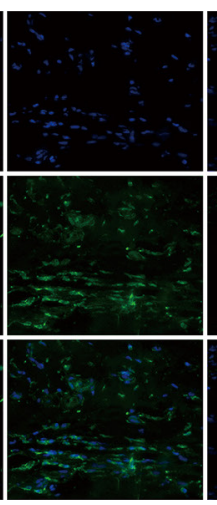

$400 \times$

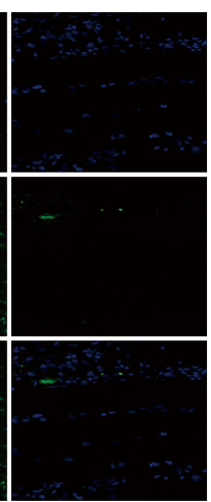

$400 \times$

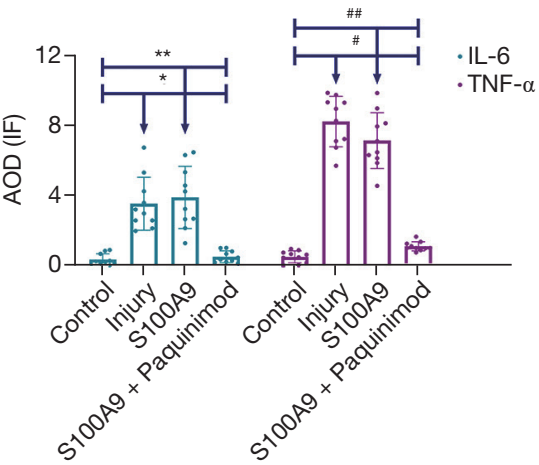

Figure 6 Expression of inflammatory factors in each group. (A) MMP-3 IF illustrated that MMP-3 was highly expressed in the Injury and S100A9 groups. Black arrow: Position of the ruptured end of Achilles tendon. (B) IL-6 IF showed that and IL-6 was highly expressed in the Injury and S100A9 groups. (C) TNF- $\alpha$ IF demonstrated that TNF- $\alpha$ exhibited a high expression in the Injury and S100A9 groups. (D) Statistical chart of AOD of MMP-3 IF expression: *, Injury-Control (or S100A9 + Paquinimod): H=-4.488 (or 3.723), P<0.05; **, S100A9Control (or S100A9 + Paquinimod): $\mathrm{H}=-3.933$ (or 3.168), $\mathrm{P}<0.05$. (E) Statistical chart of quantitative index of AOD of IL-6 and TNF- $\alpha$ IF expressions: *, Injury-Control (or S100A9 + Paquinimod): H==-4.036 (or 3.309), P<0.05; **, S100A9-Control (or S100A9 + Paquinimod): $\mathrm{H}=-4.342$ (or 3.615), $\mathrm{P}<0.05$; ", Injury-Control (or S100A9 + Paquinimod): H=-5.126 (or 3.328), P<0.05, \#, S100A9-Control (or S100A9 + Paquinimod): $\mathrm{H}=-4.323$ (or 2.525), $\mathrm{P}<0.05$. 


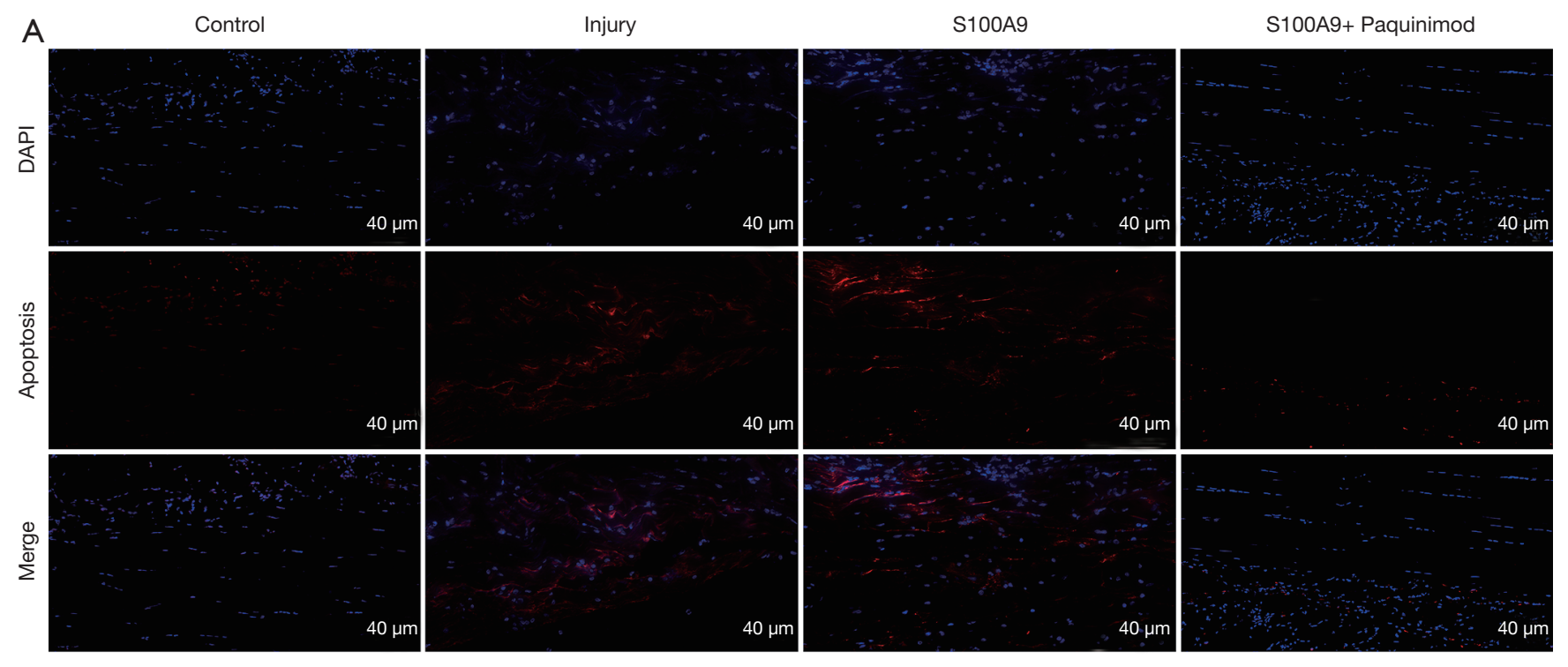

B

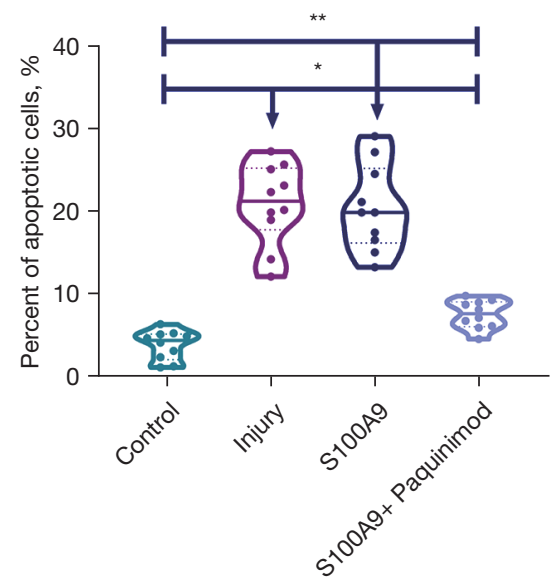

Figure 7 The apoptosis of Achilles tendon tissue. (A) TUNEL assay showed the apoptosis in the four groups, and a large number of apoptosis occurred in the Injury and S100A9 groups. (B) Statistical chart of the apoptosis rate in the four groups: The apoptosis rate in the Injury and S100A9 groups was obviously higher. *, Injury-Control (or S100A9 + Paquinimod): H=-4.753 (or 3.108), P<0.05; **, S100A9Control (or S100A9 + Paquinimod): $\mathrm{H}=-4.543$ (or 2.898), $\mathrm{P}<0.05$.

expressed in both the Achilles tendon injury model and the S100A9 injection model in rats. Meanwhile, in the Injury and S100A9 groups, tendon tissue morphology suffered from AT-like changes, collagen fibers were reconstructed, a large amount of Col-III was produced to replace Col-I, there was infiltration of inflammatory factors, and a large number of tendon cells died. However, following an injection of the S100A9 inhibitor Paquinimod, the efficiency of inducing AT-like changes decreased. Hence, it can be inferred that Alarmin S100A9 is able to induce AT and performs a vital function in the occurrence and development of AT.

The $\mathrm{S} 100$ protein, a soluble protein in brain tissues, was isolated, purified, and identified for the first time in 1965, and was named after its $100 \%$ dissolution in ammonium sulfate solution (31). It is involved in the regulation of key intracellular signaling pathways, such as cytoskeleton and cell migration, adhesion, and calcium ion stability, and can also be secreted extracellularly to regulate cell growth, differentiation, and apoptosis, as well as inducing inflammatory reactions (31-33). S100A9 has recently been shown to exert a vital effect on the pathogenesis of OA, and is highly expressed in early OA chondrocytes. Meanwhile, adding S100A9 into chondrocytes cultured in vitro prominently upregulates $A D A M T S-1,-4,-5$, and $M M P-1$, 
$-3,-13$ gene expression in chondrocytes, and significantly reduces the messenger Ribonucleic Acid expression of both Col-II and proteoglycan, which accelerates the degeneration of articular cartilage (34). van Lent et al. (35) established a mouse model of OA using the collagenase induction method, and observed marked synovitis and destruction of articular cartilage. S100A9 has also long been highly expressed in articular cartilage. Therefore, it can be speculated that Alarmin S100A9 plays an indispensable role in triggering inflammatory reactions and tissue degeneration.

Regarding tendon tissues, Zhang et al. (17) showed in animal experiments that numerous alarmins, including the S100A9 protein, exhibit high expression levels in a mouse rotator cuff tendinopathy model. In vitro experiments conducted by Crowe et al. (18) demonstrated that the S100A8/S100A9 protein is highly expressed in human supraspinatus tendinopathy and induces local inflammation, which exerts a crucial effect in the pathological process of AT. Numerous studies have indicated that inflammation is an important factor inducing AT, and tendon tissues suffering from oxidative stress or slight injury (i.e., athletes' Achilles tendon tissues under high intensity load) will inevitably trigger the production of a large number of alarmins (36-38).

In this study, $\mathrm{HE}$ and $\mathrm{SOFG}$ staining revealed the histomorphological differences between the Achilles tendon tissues in the four groups (Figure 2). It was observed that when the Achilles tendons of the rats were ruptured, the collagen fibers at the ruptured end were extremely disorganized and partially ruptured, with cartilage matrixlike changes (SOFG showed red tissues). Zhang et al. (29) demonstrated that this might be related to the heterotopic ossification of the Achilles tendon in the latter stage. When the S100A9 protein was injected into the Achilles tendon tissues, tendinopathy-like morphological changes also appeared. Following the injection of the S100A9 protein inhibitor Paquinimod, the morphological changes within the Achilles tendon tissues and normal Achilles tendon tissues were intact. Therefore, in light of this morphology, it is speculated that the S100A9 protein can induce AT.

Furthermore, this study revealed that in the Injury group, a large amount of the S100A9 protein was highly expressed when the Achilles tendon was ruptured, even when the S100A9 protein was not injected (Figure 4). Therefore, it can be further inferred that the S100A9 protein, as a type of alarmin, is expressed and released in large quantities when oxidative stress or micro-injury occurs in the body, which is a key inflammatory mediator in the stress state of the body and a vital influencing factor for inducing AT. This is also consistent with previous research results $(16,18)$.

Collagen fiber remodeling is a crucial feature of AT (4). Normal Achilles tendon tissues are composed of massive amounts of Col-I, with relatively little Col-II. In this study, it was found that when the Achilles tendon broke and the S100A9 protein was injected, numerous fresh and immature collagen fibers were produced (Figure 3). Meanwhile, IF and SR staining results (Figure 5) revealed that Col-I levels dropped dramatically in Achilles tendon tissues, but a large amount of Col-III was produced. Therefore, it can be inferred that the large amounts of fresh and immature collagen fibers observed in the Masson's stained tissue was Col-III. Accordingly, it can be seen that the S100A9 protein induces collagen fiber remodeling, thus triggering AT.

Inflammation, collagen remodeling, and ECM remodeling are significant characteristics of a changing extracellular microenvironment (37). The change of ECM is a vital factor influencing the occurrence and development of AT or tendon healing and repair, in which MMP-3 plays an indispensable role. A previous study indicated that MMP3 exerts a key effect in the pathological process of chronic AT through its impact on ECM remodeling, and can also increase the risk of chronic AT (38). In the present research, a large amount of MMP-3 was produced and massive levels of IL- 6 and TNF- $\alpha$ were released in both the Injury and S100A9 groups (Figure 6). Hence, it can be inferred that the S100A9 protein is able to induce changes in the ECM and give rise to strong inflammatory reactions, thus affecting the fibrogenesis process of tendon cells, further inducing the reconstruction of collagen fibers, thereby resulting in tendinopathy-like changes.

Apoptosis is an obvious pathological change of AT. Tendon tissues lose self-repair or regeneration capacity, especially in cases of apoptosis of numerous tendon cells or tendon stem cells that undergo apoptosis, thus inducing AT. As such, tendon tissues lose important physiological functions due to degeneration, resulting in fracture or instability $(4,9,39)$. The above-mentioned collagen fiber reconstruction is probably a process of tendon tissue selfrepair involving tendon cells or tendon stem cells (9). Hence, maintaining the activity of tendon cells or tendon stem cells and preventing apoptosis are crucial for the prevention of AT and AT deterioration. In the present study, when the Achilles tendon tissues were ruptured (Injury group), a number of cells died at the ruptured end, which might be a vital factor influencing the healing of 
the ruptured end. Following the injection of the S100A9 protein into the Achilles tendon tissues, the Achilles tendon tissues exhibited widespread cell apoptosis, which was alleviated following the injection of Paquinimod, an inhibitor of the S100A9 protein (Figure 7). Therefore, it can be inferred that the S100A9 protein exerts a vital effect in triggering tendon cell apoptosis, and a large amount of the S100A9 protein is therefore highly expressed after Achilles tendon rupture. Moreover, the apoptosis of cells in the Achilles tendon ruptured end may also be closely associated with S100A9 protein production.

Despite the interesting findings, there are some shortcomings in this study that should be noted. Firstly, there was no time control group, and the occurrence and development processes of AT over time was not studied. Multiple previous studies have shown that when inducing an animal model of AT, the peak of occurrence and development of AT occurs within approximately 1 week, at which point the pathological and histological changes in tendon tissues are very obvious $(17,29)$; therefore, in-depth research was conducted at the 1 week stage. Secondly, an Achilles tendon rupture + S100A9 inhibitor Paquinimod group was not included. This study mainly aimed to investigate whether S100A9 can induce AT and tendinopathy-like changes, so as to assess the correlation between S100A9 protein and AT. The results of this study confirmed this speculation; thus, an Achilles tendon rupture + S100A9 inhibitor Paquinimod group was not included. In future, more time points and groups need to be established for analysis and research, in order to further explore the association between the S100A9 protein and AT, as well as the occurrence and development mechanisms of AT.

\section{Conclusions}

Alarmin S100A9 is able to trigger AT, gives rise to corresponding tendinopathy-like morphological changes and local inflammatory reactions, and induces collagen fiber remodeling and tendon cell apoptosis. These results support future research into the pathogenesis and development of AT, and provide a potential new direction and novel target for its clinical treatment.

\section{Acknowledgments}

Funding: This study was funded by the National Natural Science Foundation of China (81972095), the Project of Shanghai Science and Technology Commission
(19441901702), and the Project of Shanghai Health Commission (20194Y0385).

\section{Footnote}

Reporting Checklist: The authors have completed the ARRIVE reporting checklist. Available at https://dx.doi. org/10.21037/atm-21-5945

Data Sharing Statement: Available at https://dx.doi. org/10.21037/atm-21-5945

Conflicts of Interest: All authors have completed the ICMJE uniform disclosure form (available at https://dx.doi. org/10.21037/atm-21-5945). The authors have no conflicts of interest to declare.

Ethical Statement: The authors are accountable for all aspects of the work in ensuring that questions related to the accuracy or integrity of any part of the work are appropriately investigated and resolved. Experiments were performed under a project license (SHSY-IECBG/05.08/04.0) granted by the Ethics Committee of Shanghai Tenth People's Hospital (Tenth People's Hospital Affiliated to Tongji University) Medical Ethics Committee and in compliance with the institutional guidelines for the care and use of animals.

Open Access Statement: This is an Open Access article distributed in accordance with the Creative Commons Attribution-NonCommercial-NoDerivs 4.0 International License (CC BY-NC-ND 4.0), which permits the noncommercial replication and distribution of the article with the strict proviso that no changes or edits are made and the original work is properly cited (including links to both the formal publication through the relevant DOI and the license). See: https://creativecommons.org/licenses/by-nc-nd/4.0/.

\section{References}

1. Longo UG, Ronga M, Maffulli N. Achilles Tendinopathy. Sports Med Arthrosc Rev 2018;26:16-30.

2. D'Addona A, Maffulli N, Formisano S, et al. Inflammation in tendinopathy. Surgeon 2017;15:297-302.

3. Li HY, Hua YH. Achilles Tendinopathy: Current Concepts about the Basic Science and Clinical Treatments. Biomed Res Int 2016;2016:6492597.

4. Abate M, Silbernagel KG, Siljeholm C, et al. Pathogenesis 
of tendinopathies: inflammation or degeneration? Arthritis Res Ther 2009;11:235.

5. Cook JL, Purdam CR. Is tendon pathology a continuum? A pathology model to explain the clinical presentation of load-induced tendinopathy. Br J Sports Med 2009;43:409-16.

6. Maffulli N, Khan KM, Puddu G. Overuse tendon conditions: time to change a confusing terminology. Arthroscopy 1998;14:840-3.

7. Cardoso TB, Pizzari T, Kinsella R, et al. Current trends in tendinopathy management. Best Pract Res Clin Rheumatol 2019;33:122-40.

8. Millar NL, Murrell GA, McInnes IB. Inflammatory mechanisms in tendinopathy - towards translation. Nat Rev Rheumatol 2017;13:110-22.

9. Sharma P, Maffulli N. Biology of tendon injury: healing, modeling and remodeling. J Musculoskelet Neuronal Interact 2006;6:181-90.

10. Voronov E, Dinarello CA, Apte RN. Interleukin-1 $\alpha$ as an intracellular alarmin in cancer biology. Semin Immunol 2018;38:3-14.

11. Andersson U, Yang H, Harris H. High-mobility group box 1 protein (HMGB1) operates as an alarmin outside as well as inside cells. Semin Immunol 2018;38:40-8.

12. Vogl T, Stratis A, Wixler V, et al. Autoinhibitory regulation of S100A8/S100A9 alarmin activity locally restricts sterile inflammation. J Clin Invest 2018;128:1852-66.

13. Taha EA, Ono K, Eguchi T. Roles of Extracellular HSPs as Biomarkers in Immune Surveillance and Immune Evasion. Int J Mol Sci 2019;20:4588.

14. van den Bosch MH, Blom AB, Schelbergen RF, et al. Alarmin S100A9 Induces Proinflammatory and Catabolic Effects Predominantly in the M1 Macrophages of Human Osteoarthritic Synovium. J Rheumatol 2016;43:1874-84.

15. Ruan G, Xu J, Wang K, et al. Associations between serum S100A8/S100A9 and knee symptoms, joint structures and cartilage enzymes in patients with knee osteoarthritis. Osteoarthritis Cartilage 2019;27:99-105.

16. Cremers NAJ, van den Bosch MHJ, van Dalen S, et al. S100A8/A9 increases the mobilization of pro-inflammatory Ly6Chigh monocytes to the synovium during experimental osteoarthritis. Arthritis Res Ther 2017;19:217.

17. Zhang Y, Deng XH, Lebaschi AH, et al. Expression of alarmins in a murine rotator cuff tendinopathy model. J Orthop Res 2020;38:2513-20.

18. Crowe LAN, McLean M, Kitson SM, et al. S100A8 \& S100A9: Alarmin mediated inflammation in tendinopathy. Sci Rep 2019;9:1463.
19. Schelbergen RF, Geven EJ, van den Bosch MH, et al. Prophylactic treatment with S100A9 inhibitor paquinimod reduces pathology in experimental collagenase-induced osteoarthritis. Ann Rheum Dis 2015;74:2254-8.

20. Wu PT, Su WR, Li CL, et al. Inhibition of CD44 induces apoptosis, inflammation, and matrix metalloproteinase expression in tendinopathy. J Biol Chem 2019;294:20177-84.

21. Gergye CH, Zhao Y, Moore RH, et al. A Comparison of Ketamine or Etomidate Combined with Xylazine for Intraperitoneal Anesthesia in Four Mouse Strains. J Am Assoc Lab Anim Sci 2020;59:519-30.

22. Tahvili S, Törngren M, Holmberg D, et al. Paquinimod prevents development of diabetes in the non-obese diabetic (NOD) mouse. PLoS One 2018;13:e0196598.

23. Shi L, Zhao Y, Fei C, et al. Cellular senescence induced by S100A9 in mesenchymal stromal cells through NLRP3 inflammasome activation. Aging (Albany NY) 2019;11:9626-42.

24. Yang H, Lee HJ, Lee YG, et al. Integrity of the Untorn Articular-Sided Tendon in Bursal-Sided Partial-Thickness Rotator Cuff Tear: A Comparative Study of Apoptotic Activity in Torn and Untorn Layers. Am J Sports Med 2018;46:2478-85.

25. Chen G, Chen P, You T, et al. Allogenic TendonAutologous Cartilage Cells Transplantation Enhances Adhesive/Growth Ability and Promotes Chondrogenesis in a Rabbit Model of Glenoid Labrum Damage. Ann Transplant 2019;24:532-40.

26. Zhang Y, He F, Chen Z, et al. Melatonin modulates IL$1 \beta$-induced extracellular matrix remodeling in human nucleus pulposus cells and attenuates rat intervertebral disc degeneration and inflammation. Aging (Albany NY) 2019;11:10499-512.

27. Wang Y, He G, Tang H, et al. Aspirin promotes tenogenic differentiation of tendon stem cells and facilitates tendinopathy healing through regulating the GDF7/Smad1/5 signaling pathway. J Cell Physiol 2020;235:4778-89.

28. Bei M, Tian F, Liu N, et al. A Novel Rat Model of Patellofemoral Osteoarthritis Due to Patella Baja, or LowLying Patella. Med Sci Monit 2019;25:2702-17.

29. Zhang Q, Zhang Y, Yan M, et al. ßig-h3 enhances chondrogenesis via promoting mesenchymal condensation in rat Achilles tendon heterotopic ossification model. Aging (Albany NY) 2020;12:7030-41.

30. Mao H, Su P, Qiu W, et al. The use of Masson's trichrome staining, second harmonic imaging and two-photon 
excited fluorescence of collagen in distinguishing intestinal tuberculosis from Crohn's disease. Colorectal Dis 2016;18:1172-8.

31. Donato R. Functional roles of S100 proteins, calciumbinding proteins of the EF-hand type. Biochim Biophys Acta 1999;1450:191-231.

32. Donato R. S100: a multigenic family of calciummodulated proteins of the EF-hand type with intracellular and extracellular functional roles. Int J Biochem Cell Biol 2001;33:637-68.

33. Zimmer DB, Cornwall EH, Landar A, et al. The S100 protein family: history, function, and expression. Brain Res Bull 1995;37:417-29.

34. Zreiqat H, Belluoccio D, Smith MM, et al. S100A8 and S100A9 in experimental osteoarthritis. Arthritis Res Ther 2010;12:R16.

35. van Lent PL, Blom AB, Schelbergen RF, et al. Active involvement of alarmins S100A8 and S100A9 in the regulation of synovial activation and joint destruction

Cite this article as: Zhao BA, Li J, Xue C, Li J, Ge HA, Cheng B, Su QH. Role of the Alarmin S100A9 protein in inducing Achilles tendinopathy in rats. Ann Transl Med 2021;9(22):1698. doi: 10.21037/atm-21-5945 during mouse and human osteoarthritis. Arthritis Rheum 2012;64:1466-76.

36. Kendal AR, Layton T, Al-Mossawi H, et al. Multi-omic single cell analysis resolves novel stromal cell populations in healthy and diseased human tendon. Sci Rep 2020;10:13939.

37. Andarawis-Puri N, Flatow EL. Promoting effective tendon healing and remodeling. J Orthop Res 2018;36:3115-24.

38. Nie G, Wen X, Liang X, et al. Additional evidence supports association of common genetic variants in MMP3 and TIMP2 with increased risk of chronic Achilles tendinopathy susceptibility. J Sci Med Sport 2019;22:1074-8.

39. Chen S, Jiang S, Zheng W, et al. RelA/p65 inhibition prevents tendon adhesion by modulating inflammation, cell proliferation, and apoptosis. Cell Death Dis 2017;8:e2710.

(English Language Editor: A. Kassem) 